\title{
Multi-Scale Modelling of Cerebral Blood Flow and its Control
}

\author{
Stephen Payne \\ Institute of Biomedical Engineering, Department of Engineering Science \\ University of Oxford \\ United Kingdom
}

The human brain requires a continuous flow of blood in order to maintain the tight balance between supply and demand of nutrients (primarily oxygen and glucose). Cerebral blood flow is thus very tightly regulated, with impaired control being found in a wide range of cerebrovascular diseases. With the increasing prevalence of diseases such as dementia, and a growing appreciation of the importance of blood flow in these diseases, there is an urgent need to understand better how the brain controls blood flow across a wide range of length scales. In this talk, I will examine how these processes can be modelled, covering vessels from the large arteries to the capillary bed, and describe work under way to link these models directly to clinical imaging, so that we can gain a more direct measurement of cerebral blood flow control. I will also discuss ongoing work on translating this into clinical practice for the improved management of stroke patients and its potential implications in dementia. 